\title{
Acoustical properties of molecular interactions in 1-propanol, 1-butanol and 1-pentanol with tetrahydrofuran in cyclohexanone at various temperatures.
}

\author{
Ubagaramary $D^{1,2^{*}}$, Muthu Vijayan Enoch $I^{3}$, Mullainathan $S^{4}$, Kesavaswamy \\ ${ }^{1}$ R\&D Centre, Bharathiyar University, Coimbatore, Tamil Nadu, India \\ ${ }^{2}$ Adhiyamaan College of Engineering, Hosur, India \\ ${ }^{3}$ Department of Chemistry, Karunya University, Coimbatore, Tamil Nadu, India \\ ${ }^{4}$ Department of Physics, Thiru. Vi. Ka. Government Arts College, Thiruvarur, Tamil Nadu, India \\ ${ }^{5}$ Department of Physics, Ramakrishna Engineering College, Coimbatore, Tamil Nadu, India
}

\begin{abstract}
The functions of ultrasonic velocity, density and viscosity for ternary liquid mixtures of 1-proponol, 1butanol and 1-pentanol with tetra hydro furan are determined by $303.15 \mathrm{~K}-313.15 \mathrm{~K}$. This data is used to calculate various parameters like the excess free volume, excess internal pressure and Gibb's free energy, which is used to discuss molecular interactions in the ternary liquid mixtures.
\end{abstract}

Keywords: Ternary mixtures, Ultrasonic velocity, Excess free volume, Viscosity predictive models.

Accepted on August 1, 2018

\section{Introduction}

In current years, ultrasonic speeds are effectively used in the energetic environment of molecular interactions in pure liquids and liquid compounds. Ultrasonic technique shows the physical chemical behaviour of liquids as part of a pointer to comfort. The molecular coordination between liquid compounds components is based on analysis concentrations. Mixed compounds are designed for spectroscopic technique. Spectroscopic methods were very common for these exercises. It is responsible for the nature properties of molecular contact in the body environment and liquid compounds. The ultrasonic speed of a fluid is attached.

Attractive forces between polar molecules form from the middle (water), not from direct contact between membrane molecules. Inactive participants in hydrophobic materials hydrophilic events. The liquid hydrocarbon is similar to the hydrocarbon molecules of the molecular interaction of a hydrocarbon with water molecules in aqueous solution.

Hydrophilic effect is an indirect result of strong directional relationships between water molecules and those interactions. The hydrophilic effect consists entirely of water; It is the result of the distinctive molecular structure of water and the unique harmonious properties of water. We can understand molecular and thermodynamic explanations for hydrophobic effect.

The molecular exchanges in liquid water are very complex, surprising and influential, and we have to pay special attention to them. Biology and biochemistry are taking place in complex acupuncture contexts. Living organisms are $80 \%$ water weight.
The life we know on earth is entirely dependent on water. The main water source of structures and properties of cellular assemblies and argons and biochemical reactions. Water is a reactive chemical and biologic (Hydrolysis and diabetes/ suppression), some of the most direct and critical participants of central and global reactions. Bio-polymers are collected by diabetes/allergy, all of which are broken down by hydrolysis.

Molecular interaction is associated with 1-alkanol hybrids, which are one of the most unique components since the alkonols group is very polar, because it is deeply with other groups that attraction of polar ends. Tetrahydrofuran THF is a heterocyclic organic compound formation $\left(\left(\mathrm{CH}_{2}\right)_{4} \mathrm{O}\right)$. It is a colourless low viscosity fluid with a perfume compared to diethyl ether [1-4]. It is one of the highest polar ethers. THF is the equivalent of the aromatic composite furan entirely hydrogenated. The most widely used industrial process involves the acid-catalysed dehydration of 1, 4 butane diol. Ashland/ISP route is one the biggest producers of this chemical. The method is similar to the production of ethanol. Du developed a process for producing THF by oxidizing nbutane to crude malefic anhydride, followed by catalytic hydrogenation. A third major industrial route of hydroformylation alkyl alcohol followed by hydrogenation to entail the butanediol.

The structure of tetrahydrofuran is given by Figure 1 .

Cyclohexanone is heterocyclic compound. It is highly inert towards ordinary temperature at an electrophile or nucleophile. Non-polar being cyclohexanone is not expected to be involved 
in any strong interaction with the other components of the mixture $[5,6]$. However, types of dispersive interactions between them are possible. The structure of cyclohexanone is given by Figure 2.

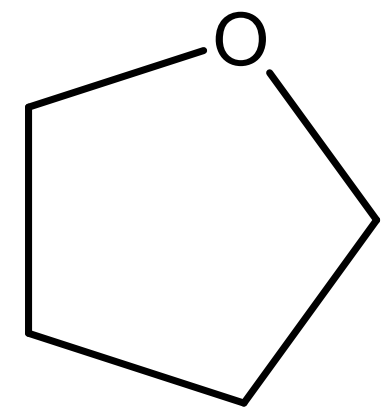

Figure 1. Structure of tetrahydrofuran.

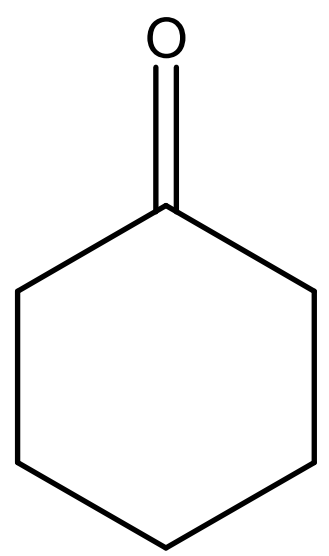

Figure 2. Structure of cyclohexanone.

Therefore, tentative studies have been taken by the authors to distinguish between the system of $\mathrm{THF}+$ cyclohexanone +1 propanol, 1-butanol and1-pentanol over ultrasonic velocity extents at $303.15 \mathrm{~K}-313.15 \mathrm{~K}$. The determination of molecular interactions in this study is used to illustrate the effect of these classifications and consequently to regulate the length of the chain 1-alkanols. It says that in all the mixtures mole fraction increase in temperature due to decrease in thermal agitation.

The structure of 1-propanol, 1-butanol and 1-pentanol are given by Figure 3.

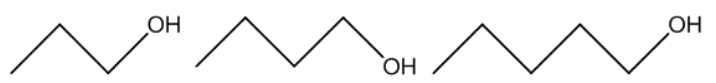

Figure 3. Structures of 1-propanol, 1-butanol and 1-pentanol.

\section{Materials and Methods}

The mixtures of mole fraction in various concentrations were prepared by taking spectroscopic analytical reagent grade and reagent grade chemicals with minimum assay of $99.9 \%$ and obtained from E Merck Ltd. (India). In all the mixtures, the mole fraction of the second component, cyclohexanone $\left(X_{2}=0.4\right)$, was kept fixed while the mole fractions of the remaining two (THF $\mathrm{X}_{1}$ and 1-propanol $\mathrm{X}_{3}$ ) were varied from 0.0 to 0.8 , so as to have different concentration of the mixture. There is nothing significant in fixing the mole fraction of the second component at 0.4 . The density, viscosity, and ultrasonic velocity were measured as a function of concentration of the liquid ternary mixture at $303.15 \mathrm{~K}-313.15 \mathrm{~K}$.

\section{Velocity measurement}

Ultrasonic velocity measurements were made using an ultrasonic interferometer (Model M-81, supplied by M/S Mittal Enterprises, New Delhi), having the frequency of $3 \mathrm{MHz}$ has been used for velocity measurements at $\mathrm{T}=303.15 \mathrm{~K}-313.15 \mathrm{~K}$ with the accuracy of $\pm 0.1 \mathrm{~m} \cdot \mathrm{s}^{-1}$. The measuring cell is a specially designed interferometer of double-walled vessel with provision for temperature constancy. An electronically operated digital constant temperature bath (Model SSI-03 Spl, supplied by M/S Mittal Enterprises, New Delhi), operating in the temperature range of $-10^{\circ} \mathrm{C}$ to $85^{\circ} \mathrm{C}$ with an accuracy of \pm $0.1^{\circ} \mathrm{C}$ has been used to circulate water through the outer jacket of the double-walled measuring cell containing the experimental liquid.

$U=n \lambda \rightarrow(1)$

Where $n$ is the frequency of the ultrasonic waves. $\lambda$ is the wavelength.

\section{Density measurement}

The mixture of densities was measured using a $10 \mathrm{ml}$ specific gravity bottle method by measurement with an accuracy of \pm relative specific gravity of $0.01 \mathrm{kgm}^{-3}$. The mixture bottle was immersed in the temperature-controlled experiment with the bath water. The weight of the sample was measured using an electronic digital balance with an accuracy of $\pm 0.1 \mathrm{mg}$ (model: SHIMADZUAX-200, Kyoto, Japan).

$\rho_{\mathrm{s}}=\left(W_{\mathrm{S}} / W_{\mathrm{W}}\right) \times \rho_{\mathrm{W}} \rightarrow(2)$

$\rho_{\mathrm{W}}$ is the density of water.

$\rho_{\mathrm{S}}$ is the density of solution.

$W_{\mathrm{S}}$ is the weight of solution.

$W_{\mathrm{w}}$ is the weight of water.

\section{Viscosity measurement}

Measurements Ostwald viscometer $10 \mathrm{ml} \pm 0.01 \mathrm{Ns}^{-1} \mathrm{~m}^{-2}$ was used for measuring viscosity of flowing time with the accuracy of \pm 0.1 was determined using a digital precision racer stop watch.

$\eta_{\mathrm{s}} \rightarrow(3)$

Where

$\eta_{\mathrm{s}}$-Viscosity of the solution,

$\eta_{0}$-Viscosity of water.

$\rho$-Density of the solution. 
Acoustical properties of molecular interactions in 1-propanol, 1-butanol and 1-pentanol with tetrahydrofuran in cyclohexanone at various temperatures

$\rho_{0}$-Density of water.

$t$-The time flow for the solution.

$t_{0}$-The time flow for water.

\section{Results and Discussion}

The density $(\rho)$, viscosity $(\eta)$ and the ultrasonic velocity $(U)$ of pure liquids and three ternary liquid systems at 303.15

Table 1. Measured values of density $(\rho)$ of the pure liquids at $303.15 \mathrm{~K}$
K-313.15 $\mathrm{K}$ are tabulated in Table 1 and Figure 1 and the additional standard viscosity, adiabatic compressibility, free length free module, internal pressure, energy, free volume, internal pressure, Gibb's energy free energy and Gruenberg interaction parameter ' $d$ ' are also listed in the Table 2.

\begin{tabular}{llll}
\hline & Density $(\rho) \mathbf{~ k g m}^{-3}$ & & Reference \\
\cline { 2 - 3 } Components & Observed values & Literature values & Rathnam et al. (2013) \\
\hline Tetrahydrofuran (THF) & 870 & 877.1 & Nayeem et al. (2014) \\
\hline Cyclohexanone & 946.4 & 940 & Palani et al. (2008) \\
\hline 1-propanol & 798 & 801.1 & Nayeem et al. (2014) \\
\hline 1-butanol & 800 & 805 & Palani et al. (2008) \\
\hline 1-pentanol & 802 & 809 &
\end{tabular}

Table 2. Values of density $(\rho)$ viscosity $(\eta)$ and ultrasonic velocity $(U)$ for $T H F\left(X_{1}\right)+$ cyclohexanone $\left(X_{2}\right)+1-$ propanol $\left(X_{3}\right)$ and THF $\left(X_{1}\right)+$ cyclohexanone $\left(X_{2}\right)+1$-pentanol $\left(X_{3}\right)$ at THF $\left(X_{1}\right)+$ cyclohexanone $\left(X_{2}\right)+1$-pentanol $\left(X_{3}\right)$ at $303.15 \mathrm{~K}$-313.15 K.

\begin{tabular}{|c|c|c|c|c|c|c|c|c|c|}
\hline \multirow[t]{2}{*}{ Mole fraction $\left(X_{3}\right)$} & \multicolumn{3}{|c|}{ Density $(\rho) \mathrm{Kgm}^{-3}$} & \multicolumn{3}{|c|}{ Viscosity $(\eta) \times 10^{3} \mathrm{Nsm}^{-2}$} & \multicolumn{3}{|c|}{ Ultrasonic velocity $(\mathrm{U}) \mathrm{ms}^{-1}$} \\
\hline & $303.15 \mathrm{~K}$ & $308.15 \mathrm{~K}$ & $313.15 \mathrm{~K}$ & $303.15 \mathrm{~K}$ & $308.15 \mathrm{~K}$ & $313.15 \mathrm{~K}$ & $303.15 \mathrm{~K}$ & $308.15 \mathrm{~K}$ & $313.15 \mathrm{~K}$ \\
\hline \multicolumn{10}{|c|}{ THF $\left(X_{1}\right)+$ cyclohexanone $\left(X_{2}\right)+1$-propanol $\left(X_{3}\right)$ at $303.15-313.15 \mathrm{~K}$. } \\
\hline 0 & 758.8 & 755.7 & 752.6 & 0.6174 & 0.5687 & 0.5613 & 1202.3 & 1192.4 & 0.5613 \\
\hline 0.1444 & 751 & 755.6 & 749.1 & 0.6477 & 0.5665 & 0.5244 & 1200.4 & 1194.3 & 0.5244 \\
\hline 0.2119 & 750.5 & 740.6 & 748.8 & 0.6534 & 0.5669 & 0.5276 & 1198.6 & 1196 & 0.5276 \\
\hline 0.3442 & 739.3 & 738.4 & 735.9 & 0.67 & 0.567 & 0.5356 & 1197.2 & 1197.2 & 0.5356 \\
\hline 0.4948 & 730.3 & 734.1 & 730.4 & 0.6823 & 0.5679 & 0.5413 & 1196.3 & 1199.7 & 0.5413 \\
\hline 0.5484 & 729.9 & 732.6 & 729.9 & 0.6914 & 0.6727 & 0.5832 & 1195.6 & 1200.7 & 0.5832 \\
\hline 0.6086 & 725.2 & 731.6 & 724.7 & 0.7245 & 0.6733 & 0.6099 & 1195.8 & 1202.2 & 0.6099 \\
\hline 0.7447 & 723.1 & 730.3 & 713.1 & 0.7459 & 0.7564 & 0.6658 & 1195 & 1209.3 & 0.6658 \\
\hline 0.8 & 722.3 & 729.8 & 704.7 & 0.7632 & 0.7638 & 0.6774 & 1194.8 & 1218.2 & 0.6774 \\
\hline \multicolumn{10}{|c|}{ THF $\left(\mathrm{X}_{1}\right)+$ cyclohexanone $\left(\mathrm{X}_{2}\right)+1$-butanol $\left(\mathrm{X}_{3}\right)$ at $303.15 \mathrm{~K}-313.15 \mathrm{~K}$} \\
\hline 0 & 758.8 & 755.7 & 752.6 & 0.6174 & 0.5687 & 0.5613 & 1202.3 & 1192.4 & 1182.6 \\
\hline 0.1444 & 756.6 & 755.6 & 750.3 & 0.6346 & 0.5672 & 0.5543 & 1202.7 & 1194.3 & 1184.3 \\
\hline 0.2119 & 755.9 & 750.3 & 748.8 & 0.6583 & 0.5669 & 0.5413 & 1204.2 & 1196 & 1186.4 \\
\hline 0.3442 & 748.1 & 745.8 & 743 & 0.6845 & 0.5658 & 0.5348 & 1209.5 & 1197.9 & 1193.6 \\
\hline 0.4948 & 746 & 740.6 & 740.7 & 0.7076 & 0.5649 & 0.5244 & 1210.3 & 1199.7 & 1194.2 \\
\hline 0.5484 & 743.8 & 739.1 & 738.4 & 0.7133 & 0.6724 & 0.5689 & 1214.9 & 1200.6 & 1195.4 \\
\hline 0.6086 & 740.9 & 738.4 & 735.9 & 0.7272 & 0.6733 & 0.6099 & 1218.2 & 1202.2 & 1196.3 \\
\hline 0.7447 & 736.5 & 730.5 & 723.7 & 0.9528 & 0.7865 & 0.6321 & 1220.8 & 1210.4 & 1198.2 \\
\hline 0.8 & 732.8 & 729.3 & 725.1 & 1.0138 & 0.7638 & 0.6774 & 1224.6 & 1218.2 & 1202.2 \\
\hline
\end{tabular}




\begin{tabular}{llllllllll}
\hline THF $\left(\mathrm{X}_{1}\right)$ +cyclohexanone $\left(\mathrm{X}_{2}\right)+1$-pentnol $\left(\mathrm{X}_{3}\right)$ at $303.15 \mathrm{~K}-313.15 \mathrm{~K}$ \\
\hline 0 & 758.8 & 755.7 & 752.6 & 0.6174 & 0.5687 & 0.5613 & 1202.3 & 1192.4 & 1182.6 \\
\hline 0.1444 & 751 & 755.6 & 749.1 & 0.6477 & 0.5665 & 0.5244 & 1200.4 & 1194.3 & 1184.3 \\
\hline 0.2119 & 750.5 & 740.6 & 748.8 & 0.6534 & 0.5669 & 0.5276 & 1198.6 & 1196 & 1186.4 \\
\hline 0.3442 & 739.3 & 738.4 & 735.9 & 0.67 & 0.567 & 0.5356 & 1197.2 & 1197.2 & 1190.7 \\
\hline 0.4948 & 730.3 & 734.1 & 730.4 & 0.6823 & 0.5679 & 0.5413 & 1196.3 & 1199.7 & 1194.2 \\
\hline 0.5484 & 729.9 & 732.6 & 729.9 & 0.6914 & 0.6727 & 0.5832 & 1195.6 & 1200.7 & 1196.3 \\
\hline 0.6086 & 725.2 & 731.6 & 724.7 & 0.7245 & 0.6733 & 0.6099 & 1195.8 & 1202.2 & 1198.2 \\
\hline 0.7447 & 723.1 & 730.3 & 713.1 & 0.7459 & 0.7564 & 0.6658 & 1195 & 1209.3 & 1200.7 \\
\hline 0.8 & 722.3 & 729.8 & 704.7 & 0.7632 & 0.7638 & 0.6774 & 1194.8 & 1218.2 & 1202.2 \\
\hline
\end{tabular}

1-propanol and as THF and cyclohexanone at 303.15 K-313.15 $\mathrm{K} 1$-butanol in the mixture having a mole fraction dissimilarity additional acoustic parameters [7,8]. Higher thermodynamic properties of liquid mixtures of the molecular interaction between molecules in the direction established to be the most elegant. Depending on the strength of the interaction between different molecules identified and due to the high level of sign and size of irregularity of excess of parameters.

Table 3 shows that the Gibbs's free energy values may be worthy of significant interactions such as hydrogen bonding, which can lead to negative irregularities $[9,10]$.

Table 3. Excess values of internal pressure $\left(\pi_{i}^{E}\right)$, Gibb's free energy $(\Delta G)$ and Gruenberg interaction parameter for THF + cyclohexanone $\left(X_{1}\right)+1$ propanol $\left(X_{2}\right)$, THF $\left(X_{1}\right)+$ cyclohexanone +1 -butanol $\left(X_{2}\right)$ and $T H F\left(X_{1}\right)+$ cyclohexanone $\left(X_{2}\right)+1$-pentanol $\left(X_{3}\right)$ at $303.15 \mathrm{~K}-313.15 \mathrm{~K}$.

\begin{tabular}{|c|c|c|c|c|c|c|c|c|c|}
\hline \multirow[t]{2}{*}{ Mole fraction } & \multicolumn{3}{|c|}{$\pi_{i}^{E} \times 10^{7} N^{-2}$} & \multicolumn{3}{|c|}{$\Delta \mathbf{G} \times 10^{-23} \mathrm{~kJ} \mathrm{~mol}^{-1}$} & \multicolumn{3}{|l|}{ d } \\
\hline & $303.15 \mathrm{~K}$ & $308.15 \mathrm{~K}$ & $313.15 \mathrm{~K}$ & $303.15 \mathrm{~K}$ & $308.15 \mathrm{~K}$ & $313.15 \mathrm{~K}$ & $303.15 \mathrm{~K}$ & $308.15 \mathrm{~K}$ & $313.15 \mathrm{~K}$ \\
\hline \multicolumn{10}{|c|}{ THF $\left(X_{1}\right)+$ cyclohexanone $\left(X_{2}\right)+1$-propanol $\left(X_{3}\right)$ at $303.15 \mathrm{~K}-313.15 \mathrm{~K}$. } \\
\hline 0 & 0 & 0 & 0 & 0 & 0 & 0 & 0 & 0 & 0 \\
\hline 0.1444 & -0.0344 & -0.3697 & -0.367 & -2.0695 & -2.1185 & -2.1428 & -0.0135 & -0.0155 & -0.0167 \\
\hline 0.2119 & -0.0344 & -0.4044 & -0.3817 & -2.0768 & -2.1292 & -2.1526 & -0.0063 & -0.0073 & -0.0078 \\
\hline 0.3442 & -0.0355 & -0.4281 & -0.3995 & -2.0774 & -2.1415 & -2.1562 & -0.0042 & -0.005 & -0.0052 \\
\hline 0.4948 & -0.0366 & -0.4737 & -0.427 & -2.0874 & -2.1652 & -2.1718 & -0.0025 & -0.003 & -0.0032 \\
\hline 0.5484 & -0.0389 & -0.4668 & -0.4337 & -2.1025 & -2.162 & -2.1793 & -0.0017 & -0.0018 & -0.0021 \\
\hline 0.6086 & -0.0412 & -0.4849 & -0.4333 & -2.0991 & -2.1718 & -2.1772 & -0.0015 & -0.0016 & -0.0018 \\
\hline 0.7447 & -0.0408 & -0.4667 & -0.424 & -2.1003 & -2.1614 & -2.1664 & -0.0013 & -0.0013 & -0.0015 \\
\hline 0.8 & -0.041 & -0.5104 & -0.451 & -2.1119 & -2.1852 & -2.1805 & -0.001 & -0.001 & -0.0012 \\
\hline
\end{tabular}

THF $\left(X_{1}\right)+$ cyclohexanone $\left(X_{2}\right)+1$-butanol $\left(X_{3}\right)$ at $303.15 \mathrm{~K}-313.15 \mathrm{~K}$.

\begin{tabular}{|c|c|c|c|c|c|c|c|c|c|}
\hline 0 & 0 & 0 & 0 & 0 & 0 & 0 & 0 & 0 & 0 \\
\hline 0.1444 & -0.3687 & -0.3713 & -0.3458 & -2.0757 & -2.1175 & -2.2696 & -0.0138 & -0.0155 & -0.0158 \\
\hline 0.2119 & -0.3861 & -0.4005 & -0.3717 & -2.0779 & -2.1308 & -2.2737 & -0.0062 & -0.0073 & -0.0076 \\
\hline 0.3442 & -0.4045 & -0.4266 & -0.3951 & -2.076 & -2.1428 & -2.2745 & -0.0041 & -0.005 & -0.0052 \\
\hline 0.4948 & -0.4444 & -0.4735 & -0.4298 & -2.0857 & -2.1669 & -2.2776 & -0.0024 & -0.0031 & -0.0033 \\
\hline 0.5484 & -0.501 & -0.4648 & -0.4351 & -2.1014 & -2.1629 & -2.2617 & -0.0017 & -0.0018 & -0.0021 \\
\hline 0.6086 & -0.5172 & -0.4827 & -0.4246 & -2.1034 & -2.1726 & -2.2482 & -0.0015 & -0.0016 & -0.0018 \\
\hline 0.7447 & -0.4415 & -0.4547 & -0.4327 & -2.0603 & -2.1533 & -2.2382 & -0.0009 & -0.0012 & -0.0015 \\
\hline
\end{tabular}




\begin{tabular}{|c|c|c|c|c|c|c|c|c|c|}
\hline 0.8 & -0.471 & -0.5127 & -0.4369 & -2.064 & -2.1843 & -2.2255 & -0.0006 & -0.001 & -0.0012 \\
\hline \multicolumn{10}{|c|}{ THF $\left(X_{1}\right)+$ cyclohexanone $\left(X_{2}\right)+1$-pentnol $\left(X_{3}\right)$ at $303.15 \mathrm{~K}-313.15 \mathrm{~K}$. } \\
\hline 0 & 0 & 0 & 0 & 0 & 0 & 0 & 0 & 0 & 0 \\
\hline 0.1444 & 4.7215 & 4.7724 & 4.7777 & 0.1026 & 8.0776 & 8.262 & -0.0135 & -0.0155 & -0.0167 \\
\hline 0.2119 & 4.7477 & 4.6226 & 4.8167 & 9.4812 & 6.4764 & 7.0583 & -0.0063 & -0.0073 & -0.0078 \\
\hline 0.3442 & 4.6381 & 4.6233 & 4.7281 & 9.276 & 5.0575 & 6.2902 & -0.0042 & -0.005 & -0.0052 \\
\hline 0.4948 & 4.6112 & 4.627 & 4.7283 & 7.9892 & 2.3085 & 4.2359 & -0.0025 & -0.003 & -0.0032 \\
\hline 0.5484 & 4.7129 & 4.6455 & 4.7734 & 6.326 & 4.7393 & 4.3042 & -0.0017 & -0.0018 & -0.0021 \\
\hline 0.6086 & 4.6961 & 4.6641 & 4.7417 & 6.8568 & 3.6837 & 4.8919 & -0.0015 & -0.0016 & -0.0018 \\
\hline 0.7447 & 4.7024 & 4.767 & 4.6332 & 6.8257 & 5.533 & 6.6304 & -0.0013 & -0.0013 & -0.0015 \\
\hline 0.8 & 4.7952 & 4.9169 & 4.5581 & 5.6272 & 3.026 & 4.9046 & -0.001 & -0.001 & -0.0012 \\
\hline
\end{tabular}

In the current study, all structures attained high negative dispersion force $\Delta \mathrm{GE}$ Gibb shows the apparent power. In recent years, characteristics of the accumulation of positive $\triangle \mathrm{GE}$ in some ternary liquid mixture, different molecules of hydrogen bond formation in tetrahydrofuran (THF) +cyclohexanone+1-propanol, tetrahydrofuran +cyclohexanone+1-butanol and tetrahydrofuran (THF) +cyclohexanone+1-pentanol at 303.15 K-313.15 K.

The corresponding values are tabulated in the Table 2 and the increasingly negative as the quality of the system of 1propanol, 1-butanol and 1-pentanol is lacking these standard due to increasing of temperature [11-14]. Table 3 shows that the magnitude of Gruenberg and Nissan equivalent parameter (d) is in the strong interaction between the components. Standards specify different types of interactions were added. Strong interaction indicates a large positive values and small positive values for the weak interaction and no interaction refers to the large negative values. Negative values should be recognized widely, depending on the strength of the forces. The anti-hydrogen bonds present in the additional component of mixtures. In the current study, no specific interactions between unlike molecules in ternary structures, the negative values of ' $d$ ' may be attributed $[15,16]$. The decreasing values of ' $d$ ' with increasing of temperature through the interaction between the components of mixtures shows weakening.

As a measure of deviation from the ideal behaviour and the combination of excess parameters are found to be strong intermolecular interactions between molecules of the mixture.

The higher values of unlike molecules are positive for the weak interaction between molecules. Whereas, the negative excess parameters in unlike molecules may be due to the presence of dipole-dipole, dipole-induced dipole, charge transfer and hydrogen bonding whereas those of dipole-dipole, dipoleinduced dipole, charge transfer and hydrogen bonding between unlike molecules gives negative excess parameters. Ultrasonic velocity dispersion in the system that causes dispersion should contain information about the relaxation time $\tau$. Due to the structural relaxation process of relaxation time $10-12 \mathrm{~s}$, it is said that it would molecules rearranged due to the co-operative process [16-18].

The excess values of internal pressure, adiabatic compressibility and Gibbs free energy indicate that the strength of the interaction between molecules with changing temperatures do not vary much and mole fraction in the case of $\mathrm{THF}+$ cyclohexanone+1-propanol mixture, while change clearly is able to detect in THF+cycloxanone+1-butanol and 1pentanol mixture [19].

This is due to the fact that THF+cycloxanone+1-propanol there may be a large dipole in comparison to $\mathrm{THF}+$ cycloxanone +1 butanol mixture. Hence, in combination with the larger substrate interaction of THF+cycloxanone+1-propanol intermolecular interaction in the mixture containing 1-propanol in cycloexanone is large compared to that containing 1-butanol and 1-pentanol in ternary liquid mixture.

\section{Conclusion}

The validity paper which presents and conclusions drawn:

1. The net increase in the mole fraction of THF increases the strong interactions. If the medium also becomes more compact, the speed increases and the continued observance of the two systems (THF+cyclohexanone+1-butanol and THF +cyclohexanone+1-pentanol).

2. The increase in temperature due to thermal agitation, resulting in a decrease in velocity of ultrasonic mutual decreases at higher temperatures. An increase in viscosity with increase in mole fraction of THF suggests that molecular interactions are increasing in thickness of the liquid mixture and molecular size and shape of the elements that play an equally important role.

3. The VFE negative values over the full range of mole fraction may be due to the difference in size and the shape of the molecules. 
4. The adiabatic compressibility suggests that the lack of interaction between unlike molecules that have the minimum declarations.

5. Unlike molecules, weak interactions provide positive higher values, while the dipole-dipole, dipole-induced dipole, charge transfer and hydrogen bonding are unlike the molecules of negative ones [19]. From the magnitude of velocity, there is molecular interactions in the combination of THF and cyclohexanone mixture, which are linked to the keto group of oxygen atoms, resulting in a weak link between them. However, the mixture is more reactive with 1-propanool. 1propaneol hydroxyl group is bound to the sp3 hybrid carbon atom and easily removed. This shows that the reaction rate with 1-propanool is faster than 1-butanol and 1-pentanol. As the magnitude of VFE, the survival of molecular connections in the mixture is increasing:

THF+cyclohexanone+1-propanol $>$ 1-butanol $>1$-pentanol.

\section{Acknowledgement}

We are grateful to Dr. Ranganath, principal of Adhiyamaan College of engineering, Hosur-635109, to provide all facilities till the end of this study.

\section{References}

1. Sam L. Intensively dried carbon tetrachloride. J Am Chem Soc 1929; 51: 2948-2950.

2. Mehra R, Sajnami H. Acoustical studies in ternary electrolytic mixtures at $25,30,35,40$ and $45^{\circ} \mathrm{C}$ Ind $\mathrm{J}$ Pure and Appl Phys 2000; 38: 762-765.

3. Fort RJ, Moore WR. Adiabatic compressibility's of binary liquid mixtures. Trans Faraday Soc 1965; 61.

4. Kasare SB, Patdai BA. Ultrasonic studies on binary and ternary mixtures of some organic liquids. Indian J Pure Appl Phys 1987; 25.

5. Ramasamy K, Ranganathan V. ultrasonic studies on some ternary organic liquid mixtures at 303, 308 and $313 \mathrm{~K}$. Indian J Pure Appl Phys 1983; 27.

6. Spencer JN, Jeffery E, Robert C. Enthalpies of solution and transfer enthalpies. An analysis of the pure base calorimetric method for the determination of hydrogen bond enthalpies. J Phys Chem 1979; 83: 1249-1255.

7. Fort RJ, Moore WR. Viscosities of binary liquid mixtures. Trans Faraday Soc 1966; 62.

8. Pratap KC, Shankar J, Ramesh L, Gardas J. Structural and compositional effect on the acoustic and volumetric properties of ammonium based ionic liquids with water and N-methyl-2-pyrrolidone. Mol Liquids 2016; 219: 829.

9. Chanda C, Meenu S, Harish K. Interionic interactions of glycine, l-alanine, glycylglycine and phenylalanine in aqueous 1-hexyl-3-methylimidazolium chloride ionic liquid solutions at $\mathrm{T}=(288.15$ to 308.15$) \mathrm{K}$ : Volumetric, speed of sound and viscometric measurements. J Mol Liquids 2016; 218: 68-82.
10. Anil Kumar N, Neha C, Ankitaa Jyoti G, Prakash C. Physicochemical study of intermolecular interactions in 1,4-dioxane + aromatic hydrocarbons binary mixtures at different temperatures by using ultrasonic and viscometric methods. J Chem Thermodynamics 2017; 108: 145-161.

11. Shahla S, Abolghasem J. Theoretical and experimental study of the thermodynamic solubility of pioglitazone $\mathrm{HCl}$ in ethanol, $\mathrm{N}$-methyl pyrrolidone or propylene glycol +water mixtures at various temperatures. J Soln Chem 2017; 46: 139-149.

12. Vani Latha S, Little Flower G, Rayapa Reddy K, Nageswara Rao CV, Ratnakar A. Densities, ultrasonic velocities, excess properties and IR spectra of binary liquid mixtures of organic esters (ethyl lactate, some organic carbonates). J Soln Chem 2017; 46: 305-330.

13. Patricia FR, Noelia C, Elena G, Angeles D. Study of the suitability of two ammonium-based ionic liquids for the extraction of benzene from its mixtures with aliphatic hydrocarbons. Fluid Phase Equilibria 2016; 426: 17-24.

14. Fort RJ, Moore WR. Viscosities of binary liquid mixtures. Trans Faraday Soc 1966; 62.

15. Ramamoorthy K, Alwan S. Calculation of free volume from ultrasonic velocity. Current Sci 1978; 47: 334-335.

16. Treszc Zanowicz AJ, Kiyohara O, Benzon GC. Excess volumes for n-alkanols + n-alkanes IV. Binary mixtures of decan-1-ol+n-pentane, +n-hexane, +n-octane, +n-decane, and + n-hexadecane. J Chem Thermodyn 1981; 13: 253-260.

17. Umadevi M, Kesavasamy R, Rathina K, Mahalakshmi R. Studies on liquid-liquid interactions of some ternary mixtures by density, viscosity and ultrasonic speed measurements. J Mol Liquids 2016; 219: 820-828.

18. Pratap KC, Shankar J, Ramesh LG. Structural and compositional effect on the acoustic and volumetric properties of ammonium based ionic liquids with water and N-methyl-2-pyrrolidone. J Mol Liquids 2016; 219: 829-844.

19. Chanda C, Meenu S, Harsh K. Interionic interactions of glycine, 1-alanine, glycylglycine and phenylalanine in aqueous 1-hexyl-3-methylimidazolium chloride ionic liquid solutions at $\mathrm{T}=288.15 \mathrm{~K}$ to $308.15 \mathrm{~K}$ : volumetric, speed of sound and viscometric measurements. J Mol Liquids 2016; 218 : 68-82.

\section{*Correspondence to}

Ubagaramary D

R\&D Centre

Bharathiyar University

Tamil Nadu

India 